UWO-TH-06/07

\title{
On jet quenching parameters in strongly coupled non-conformal gauge theories
}

\author{
Alex Buchel \\ Department of Applied Mathematics \\ University of Western Ontario \\ London, Ontario N6A 5B7, Canada \\ Perimeter Institute for Theoretical Physics \\ Waterloo, Ontario N2J 2W9, Canada
}

\begin{abstract}
Recently Liu, Rajagopal and Wiedemann (LRW) [1] proposed a first principle, nonperturbative quantum field theoretic definition of "jet quenching parameter" $\hat{q}$ used in models of medium-induced radiative parton energy loss in nucleus-nucleus collisions at RHIC. Relating $\hat{q}$ to a short-distance behavior of a certain light-like Wilson loop, they used gauge theory-string theory correspondence to evaluate $\hat{q}$ for the strongly coupled $\mathcal{N}=4 S U\left(N_{c}\right)$ gauge theory plasma. We generalize analysis of LRW to strongly coupled non-conformal gauge theory plasma. We find that a jet quenching parameter is gauge theory specific (not universal). Furthermore, it appears it's value increases as the number of effective adjoint degrees of freedom of a gauge theory plasma increases.
\end{abstract}

May 2006 


\section{Introduction}

A characteristic feature of nuclear-nuclear collisions actively studied at RHIC is QCD plasma-induced "jet quenching" of partons produced with high transverse momentum $[5,6,7,8]$. Successful models explaining such suppression of hadronic spectra typically involve a medium-sensitive "jet quenching parameter" $\hat{q}$. $\operatorname{In}^{1}[1]$ Liu, Rajagopal and Wiedemann proposed a first principle, nonperturbative quantum field theoretic definition of $\hat{q}$. Specifically, they considered an expectation value of the light-like Wilson loop $\left\langle W^{A}(\mathcal{C})\right\rangle$ in the adjoint representation whose contour $\mathcal{C}$ is a rectangle with large extension $L^{-}$in the $x^{-}$direction and small extension $L$ in a transverse direction. Motivated by so-called dipole approximation used in jet quenching calculations [9]

$$
\left\langle W^{A}(\mathcal{C})\right\rangle \approx \exp \left[-\frac{1}{4} \hat{q} L^{-} L^{2}\right]
$$

they defined $\frac{1}{4} \hat{q}$ as the coefficient of the $L^{-} L^{2}$ term in $\ln \left\langle W^{A}(\mathcal{C})\right\rangle$ at small $L$. Quarkgluon plasma produced in nuclear-nuclear collisions at RHIC is believed to be strongly coupled. Given precise definition of $\hat{q}$ as above, LRW further argued that gauge theorystring theory correspondence of Maldacena $[10,11]$ is a suitable framework for such a computation.

Currently, we do not have a useful string theoretic description of strongly coupled QCD - one typically studies "QCD-like" large- $N_{c}$ gauge theories that allow for a weakly curved supergravity description. By far the most popular model is $\mathcal{N}=4$ $S U\left(N_{c}\right)$ supersymmetric Yang-Mills theory, which in the planar limit $\left(N_{c} \rightarrow \infty\right.$, $g_{Y M} \rightarrow 0$ with $\lambda=g_{Y M}^{2} N_{c}=$ const ), and for large values of the 't Hooft coupling $\left(\lambda \gg 1\right.$ ), is described by type IIB supergravity on $A d S_{5} \times S^{5}$ [10]. More refined examples are gauge-gravity dualities with less supersymmetries $[12,13,14,15]$, or with fundamental matter $[16,17]$. Though QCD itself is not in the universality class of these (and similar) models, one hopes that certain features of real QCD can be studied in this approximation. Relevant to study of strongly coupled gauge theory plasma, such hopes are supported by recent observation that all large- $N_{c}$ strongly coupled gauge theories that have a dual supergravity description have a universal ratio $[18,19,20]$ of shear viscosity $\eta$ to the entropy density $s$,

$$
\frac{\eta}{s}=\frac{1}{4 \pi}
$$

\footnotetext{
${ }^{1}$ Alternative approaches to describing jet quenching in hot gauge theory plasma where proposed in $[2,3]$. Potential relation between two approaches has been explored in [4].
} 
in the limit ${ }^{2} \lambda \rightarrow \infty$. Also, though neither the speed of sound waves $v_{s}$ nor the bulk viscosity $\zeta$ in non-conformal gauge theories is universal, one observes an interesting phenomenological relation $[23,24,25]$

$$
\left(v_{s}^{2}-\frac{1}{3}\right) \ll 1, \quad \frac{\zeta}{\eta} \simeq-\kappa\left(v_{s}^{2}-\frac{1}{3}\right), \quad \kappa \sim 1 .
$$

An interesting question is how the jet quenching parameter $\hat{q}$ fits into this story. LRW found that for $\mathcal{N}=4 S U\left(N_{c}\right)$ SYM at strong coupling and in the 't Hooft limit, the jet quenching parameter is

$$
\hat{q}_{\mathcal{N}=4}=\frac{\pi^{3 / 2} \Gamma\left(\frac{3}{4}\right)}{\sqrt{2} \Gamma\left(\frac{5}{4}\right)} \sqrt{\lambda} T^{3}
$$

where $T$ is the temperature of the Yang-Mills plasma. Furthermore, assuming that

$$
\hat{q}_{Q C D} \approx \hat{q}_{\mathcal{N}=4}
$$

and using a reasonable set of parameters relevant for $\mathrm{Au}-\mathrm{Au}$ collisions at $\mathrm{RHIC}$, the jet quenching parameter predicted by (1.5) appear to be somewhat small compare to the jet quenching parameter $\overline{\hat{q}}$ extracted from the experiment [1]. The authors suggested two possible explanations of the observed discrepancy:

- QCD is not a conformal gauge theory; (one way) it is related to CFT is by reducing number of adjoint degrees of freedom, thus LRW conjectured that in a process of such reduction a jet quenching parameter increases;

- alternatively, they pointed out that $\overline{\hat{q}}$ as extracted from the experiment could be misleadingly high because energy loss sources besides gluon radiation ( as the only source of energy loss assumed in evaluating $\hat{q}$ ) could be important.

In this paper, following LRW proposal [1], we study the jet quenching parameters in non-conformal gauge theories. We find that $\hat{q}$ is not universal in strongly coupled gauge theory plasma in the 't Hooft limit that allow for an effective weakly curved supergravity description. On the example of cascading gauge theory [14], we explicitly show that the jet quenching parameter increases as one goes from a confining gauge theory to a conformal gauge theory. Thus, is appears that a discrepancy between $\hat{q}_{Q C D}$ (in approximation (1.5)) and $\overline{\hat{q}}$ is likely due to additional energy loss sources for the hadronic jets besides gluon radiation.

\footnotetext{
${ }^{2}$ Leading $\frac{1}{\lambda}$ corrections to this ratio for $\mathcal{N}=4 S U\left(N_{c}\right)$ SYM were computed in [21, 22].
} 
In the next section we recall relevant facts for the cascading gauge theory [14] and the supergravity dual of it's strongly coupled plasma [26, 27, 28, 29]. In section 3, following the general suggestion of [1] we evaluate the jet quenching parameter of this cascading gauge theory $\hat{q}_{\text {cascade }}$ and compare it to $\hat{q}_{\mathcal{N}=4}$. We conclude in section 4 with a proposal how a jet quenching parameter computed in the cascading gauge theory could be adapted to real QCD.

Though our emphasis in this paper is on a cascading gauge theory, our analysis can be extended to other non-conformal gauge theory plasma, such as $\mathcal{N}=2^{*}$ plasma $[30,31]$. The jet quenching parameter in $\mathcal{N}=2^{*}$ model will be discussed elsewhere.

\section{Cascading gauge theory}

In this section we recall the relevant facts about cascading gauge theories [14] and the gravitational description of their strongly coupled deconfined plasma [26, 27, 28, 29]. In particular, we emphasize why cascading gauge theory plasma is an excellent 'probe' of the behavior of the jet quenching parameter $\hat{q}$ as one goes from QCD to conformal plasma.

\subsection{Gauge theory description}

Cascading gauge theory at a given high-energy scale resembles $\mathcal{N}=1$ supersymmetric $S U\left(K_{*}\right) \times S U\left(K_{*}+P\right)$ gauge theory with two bifundamental and two anti-fundamental chiral superfields and certain superpotential, which is quartic in superfields. Unlike ordinary quiver gauge theories, an 'effective rank' of cascading gauge theories depends on an energy scale at which the theory is probed [26, 28, 29]

$$
K_{*} \equiv K_{*}(E) \sim 2 P^{2} \ln \frac{E}{\Lambda}, \quad E \gg \Lambda
$$

At a given temperature $T$ cascading gauge theory is probed at energy scale $E \sim T$, and as $T \gg \Lambda, K_{*}(T) \gg P^{2}$. In this regime the thermal properties of the theory [28, 29] are very similar to those of the $\mathcal{N}=1 S U\left(K_{*}\right) \times S U\left(K_{*}\right)$ superconformal gauge theory of Klebanov and Witten [32], with

$$
\delta_{\text {cascade }} \equiv \frac{P^{2}}{K_{*}}
$$

being the deformation parameter. As the temperature increases, the deformation parameter $\delta_{\text {cascade }}$ decreases and the theory more and more resembles conformal gauge 
theory. The latter is probably best illustrated by the behavior of the cascading gauge theory plasma transport properties, such as the speed of sound and the bulk viscosity $[24]$

$$
\begin{aligned}
& v_{s}^{2}=\frac{1}{3}-\frac{4}{9} \delta_{\text {cascade }}+\mathcal{O}\left(\delta_{\text {cascade }}^{2}\right) \\
& \frac{\zeta}{\eta}=-2\left(v_{s}^{2}-\frac{1}{3}\right)+\mathcal{O}\left(\delta_{\text {cascade }}^{2}\right) .
\end{aligned}
$$

At small temperatures (below the strong coupling scale) the cascading gauge theory, much like real QCD, is expected to confine and undergo chiral symmetry breaking ${ }^{3}$. Thus, the temperature dependence of the ratio

$$
\rho(T)=\frac{\hat{q}_{\text {cascade }}}{\hat{q}_{K W}},
$$

as one dials up the temperature is a good indicator of what happens with the jet quenching parameter as one goes from QCD to conformal Klebanov-Witten plasma with the jet quenching parameter $\hat{q}_{K W}$.

In section 3 we explicitly evaluate $\hat{q}_{\text {cascade }}$ at high temperature and show that

$$
\frac{d \rho(T)}{d T}>0, \quad T \gg \Lambda
$$

\subsection{Supergravity dual to strongly coupled deconfined cascading gauge the- ory plasma}

Supergravity dual to deconfined cascading gauge theory plasma was studied in $[26,27$, 28, 29]. The ten-dimensional Einstein frame metric takes a form

$$
d s_{10(E)}^{2}=g_{\mu \nu} d y^{\mu} d y^{\nu}+\Omega_{1}^{2}(r) e_{\psi}^{2}+\Omega_{2}^{2}(r) \sum_{i=1}^{2}\left(e_{\theta_{i}}^{2}+e_{\phi_{i}}^{2}\right),
$$

where $r$ is the radial coordinate on $\mathcal{M}_{5}$ (greek indexes $\mu, \nu$ will run from 0 to 4 ) and the one-forms $e_{\psi}, e_{\theta_{i}}, e_{\phi_{i}}(i=1,2)$ are given by :

$$
e_{\psi}=\frac{1}{3}\left(d \psi+\sum_{i=1}^{2} \cos \theta_{i} d \phi_{i}\right), \quad e_{\theta_{i}}=\frac{1}{\sqrt{6}} d \theta_{i}, \quad e_{\phi_{i}}=\frac{1}{\sqrt{6}} \sin \theta_{i} d \phi_{i} .
$$

The five-dimensional metric can be conveniently parameterized as

$$
g_{\mu \nu} d y^{\mu} d y^{\nu}=\Omega_{1}^{-2 / 3} \Omega_{2}^{-8 / 3}\left(-c_{1}^{2} d t^{2}+c_{2}^{2} d \vec{x}^{2}+c_{3}^{2} d r^{2}\right),
$$

\footnotetext{
${ }^{3}$ This was rigorously demonstrated only at zero temperature [14].
} 
with $c_{i}=c_{i}(r)$. The metric (2.8) has a regular horizon at $c_{1}(r)=0$. Finally, the background geometry also has nontrivial 5-form and 3-form fluxes and the dilaton $\Phi(r)$. Introducing a new radial coordinate

$$
x \equiv \frac{c_{1}}{c_{2}}
$$

(the horizon is now at $x=0$ and the boundary is at $x=1$ ) and

$$
\Omega_{1}=e^{f-4 w}, \quad \Omega_{2}=e^{f+w},
$$

explicit geometry and the dilaton to leading order in $\delta_{\text {cascade }}$ are determined by

$$
\begin{aligned}
c_{2} & =\frac{a}{\left(1-x^{2}\right)^{1 / 4}}\left(1+\frac{P^{2}}{K_{*}} \xi(x)\right), \quad f=-\frac{1}{4} \ln \frac{4}{K_{*}}+\frac{P^{2}}{K_{*}} \eta(x), \\
w & =\frac{P^{2}}{K_{*}} \psi(x), \quad \Phi=\frac{P^{2}}{K_{*}} \zeta(x),
\end{aligned}
$$

where $a$ is a constant nonextremality parameter, and

$$
\begin{aligned}
\xi & =\frac{1}{12}\left(1-\ln \left(1-x^{2}\right)\right) \\
\zeta & =\frac{K_{*}}{P^{2}} \Phi_{\text {horizon }}+\frac{\pi^{2}}{12}-\frac{1}{2} \operatorname{dil} \log (\mathrm{x})+\frac{1}{2} \operatorname{dil} \log (1+\mathrm{x})-\frac{1}{2} \ln \mathrm{x} \ln (1-\mathrm{x}), \\
\eta & =-\frac{3\left(1+x^{2}\right)}{80\left(1-x^{2}\right)}\left(\operatorname{dilog}\left(1-\mathrm{x}^{2}\right)-\frac{\pi^{2}}{6}\right)+\frac{1}{20}\left(1-\ln \left(1-x^{2}\right)\right) .
\end{aligned}
$$

Furthermore, $\psi$ satisfies the linear differential equation

$$
0=\psi^{\prime \prime}+\frac{1}{x} \psi^{\prime}-\frac{3}{\left(1-x^{2}\right)^{2}} \psi-\frac{1}{10\left(1-x^{2}\right)},
$$

with the boundary condition

$$
\psi=\psi_{\text {horizon }}+\mathcal{O}\left(x^{2}\right), \quad \psi=-\frac{1}{30}\left(1-x^{2}\right)+\mathcal{O}\left(\left(1-x^{2}\right)^{3 / 2}\right),
$$

where the second boundary condition will uniquely determine $\psi_{\text {horizon }}$. In what follows we fix the dilaton so that

$$
\lim _{x \rightarrow 1_{-}} \Phi=0
$$

which leads to

$$
\Phi_{\text {horizon }}=-\frac{\pi^{2} P^{2}}{24 K_{*}}
$$

The Hawking temperature $T$ of the nonextremal solution (again to leading order in $\left.\delta_{\text {cascade }}\right)$ is given by

$$
(2 \pi T)^{2}=2^{14 / 3} a^{2} K_{*}^{-4 / 3}\left(1-\frac{P^{2}}{2 K_{*}}\right) .
$$




\section{Calculation of $\hat{q}_{\text {cascade }}$}

Following [1], the jet quenching parameter is determined from the expectation value of a certain light-like Wilson loop in the adjoint representation $\left\langle W^{A}(\mathcal{C})\right\rangle$. On the supergravity side it is easiest to evaluate the thermal expectation value of a Wilson loop in the fundamental representation $\left\langle W^{F}(\mathcal{C})\right\rangle$. In the planar limit the two expectation values are related as follows

$$
\ln \left\langle W^{A}(\mathcal{C})\right\rangle=2 \ln \left\langle W^{F}(\mathcal{C})\right\rangle .
$$

According to the gauge theory-string theory correspondence $[33,34,35,36,37],\left\langle W^{F}(\mathcal{C})\right\rangle$ is given by

$$
\left\langle W^{F}(\mathcal{C})\right\rangle=\exp [-S(\mathcal{C})],
$$

where $S$ is the extremal action (subject to a suitable subtraction) of a fundamental string worldsheet whose $r \rightarrow \infty$ boundary is the $\mathcal{C}$ contour in Minkowski space $R^{3,1}$.

Using light-cone Minkowski coordinates $(t, \vec{x})=\left(x^{ \pm}, x^{2}, x^{3}\right)$, the relevant contour $\mathcal{C}$ is then a rectangle with large extension $L^{-}$in the $x^{-}$direction and small extension $L$ along the $x^{2}$ direction. As in [1], we parameterized the surface whose action $S(\mathcal{C})$ is to be extremized by

$$
\left\{\xi^{I}(\tau, \sigma)\right\}=\left\{x^{ \pm}(\tau, \sigma), x^{2}(\tau, \sigma), x^{3}(\tau, \sigma), r(\tau, \sigma), \psi(\tau, \sigma), \theta_{i}(\tau, \sigma), \phi_{i}(\tau, \sigma)\right\},
$$

for $i=1,2$, where $\sigma^{\alpha}=(\tau, \sigma)$ describe the coordinates parameterizing the worldsheet. The Nambu-Goto action for the string worldsheet is given by

$$
S=\frac{1}{2 \pi \alpha^{\prime}} \int d \sigma d \tau \sqrt{\operatorname{det} g_{\alpha \beta}}
$$

with

$$
g_{\alpha \beta}=G_{I J} \partial_{\alpha} \xi^{I} \partial_{\beta} \xi^{J},
$$

where $G_{I J}$ is the string frame metric of the background ten-dimensional geometry. In our case

$$
\begin{aligned}
& d s_{10(\text { string })}^{2}=e^{\Phi / 2} d s_{10(E)}^{2}=G_{I J} d \xi^{I} d \xi^{J} \\
= & -\left(\hat{c}_{1}^{2}+\hat{c}_{2}^{2}\right) d x^{+} d x^{-}+\frac{1}{2}\left(\hat{c}_{2}^{2}-\hat{c}_{1}^{2}\right)\left(\left(d x^{+}\right)^{2}+\left(d x^{-}\right)^{2}\right)+\hat{c}_{2}^{2}\left(d x_{2}^{2}+d x_{3}^{2}\right)+\hat{c}_{3}^{2} d r^{2} \\
& +\hat{\Omega}_{1}^{2} e_{\psi}^{2}+\hat{\Omega}_{2}^{2} \sum_{i=1}^{2}\left(e_{\theta_{i}}^{2}+e_{\phi_{i}}^{2}\right),
\end{aligned}
$$


where

$$
\hat{c}_{i}^{2}=e^{\Phi / 2} \Omega_{1}^{-2 / 3} \Omega_{2}^{-8 / 3} c_{i}^{2}, \quad \hat{\Omega}_{j}^{2}=e^{\Phi / 2} \Omega_{j}^{2},
$$

and $i=1,2,3, j=1,2$. We will fix string worldsheet coordinates as $\tau=x^{-}$and $\sigma=x^{2}$. In the limit $L^{-} \gg L$ it is consistent to assume that (apart from $x^{-}$) $\xi^{I}=$ $\xi^{I}(\sigma)$. The symmetries of the background geometry imply that the extremal string worldsheet would lie at constant $x^{+}, x^{3}, \psi, \theta_{i}, \phi_{i}$. For the remaining bulk coordinate $r$, we implement the requirement that the world sheet has $\mathcal{C}$ as its boundary by imposing

$$
r\left( \pm \frac{L}{2}\right)=\infty
$$

Notice that such an embedding preserves a symmetry $r(\sigma)=r(-\sigma)$. The action (3.3) takes form

$$
S=\frac{\sqrt{2} L^{-}}{2 \pi \alpha^{\prime}} \int_{0}^{\frac{L}{2}} d \sigma \hat{c}_{2}^{2}\left(1-\frac{\hat{c}_{1}^{2}}{\hat{c}_{2}^{2}}\right)^{1 / 2}\left(1+\frac{\hat{c}_{3}^{2}\left(r^{\prime}\right)^{2}}{\hat{c}_{2}^{2}}\right)^{1 / 2},
$$

where $r^{\prime}=\partial_{\sigma} r$. The equation of motion for $r(\sigma)$ is then

$$
\frac{\hat{c}_{3}^{2}\left(r^{\prime}\right)^{2}}{\hat{c}_{2}^{2}}=\gamma \hat{c}_{2}^{4}\left(1-\frac{\hat{c}_{1}^{2}}{\hat{c}_{2}^{2}}\right)-1
$$

where $\gamma>0$ is an integration constant. This integration constant is determined by $L$, and as we will see shortly, small values of $L$ correspond to large values of $\gamma$. The $\sigma \leftrightarrow-\sigma$ symmetry of the string worldsheet implies that $r^{\prime}(\sigma=0)=0$. At the horizon $r=r_{h}$ of the geometry (3.5) $\hat{c}_{1}$ vanishes and the left hand side of (3.9) is manifestly positive $^{4}$. In fact, one can explicitly verify that for large enough $\gamma$ it stays positive all the way from the horizon to the boundary. Thus we conclude that at $\sigma=0$ the string worldsheet must reach the horizon (where $\hat{c}_{3}\left(r_{h}\right)=\infty$ )

$$
r(\sigma=0)=r_{h}
$$

Along with (3.9), the latter boundary condition relates $\gamma$ to the transverse width $L$ of the Wilson loop

$$
\frac{L}{2}=\int_{r_{h}}^{\infty} \frac{\hat{c}_{3} d r}{\hat{c}_{2}\left(\gamma \hat{c}_{2}^{2}\left(\hat{c}_{2}^{2}-\hat{c}_{1}^{2}\right)-1\right)^{1 / 2}} .
$$

In the case of supergravity dual to $\mathcal{N}=4$ plasma

$$
\hat{c}_{2}^{2}\left(\hat{c}_{2}^{2}-\hat{c}_{1}^{2}\right)-1
$$

\footnotetext{
${ }^{4}$ At least for sufficiently large values of $\gamma$.
} 
is a constant ${ }^{5}$. In non-conformal gauge theories it varies; moreover, the combination multiplying $\gamma$ never vanishes ${ }^{6}$. Thus we conclude from (3.11) that large values of $L$ correspond to small values of $\gamma$ (in non-conformal geometries).

Using equation of motion (3.9) we can rewrite

$$
S=\frac{\sqrt{2} L^{-}}{2 \pi \alpha^{\prime}} \int_{r_{h}}^{\infty} \frac{\gamma^{1 / 2} \hat{c}_{2}^{2}\left(\hat{c}_{2}^{2}-\hat{c}_{1}^{2}\right) \hat{c}_{3} d r}{\hat{c}_{2}\left(\gamma \hat{c}_{2}^{2}\left(\hat{c}_{2}^{2}-\hat{c}_{1}^{2}\right)-1\right)^{1 / 2}}
$$

As in [1] from (3.12) one needs to subtract the self-energy $S_{0}$ of the high energy quark and antiquark moving through the plasma:

$$
S_{0}=\frac{\sqrt{2} L^{-}}{2 \pi \alpha^{\prime}} \int_{r_{h}}^{\infty} d r \sqrt{G_{--} G_{r r}}=\frac{\sqrt{2} L^{-}}{2 \pi \alpha^{\prime}} \int_{r_{h}}^{\infty} \hat{c}_{3}\left(\hat{c}_{2}^{2}-\hat{c}_{1}^{2}\right)^{1 / 2} d r .
$$

The resulting $S_{I}=S-S_{0}$ is the subtracted extremal action to be used in (3.2). Recall that we are interested in the expectation value of the thermal Wilson loop as $L \rightarrow 0$. We argued above that in that limit $\gamma \rightarrow \infty$, thus, rather explicitly, to leading order in $\gamma$ we have

$$
S_{I}=\frac{\sqrt{2} L^{-}}{2 \pi \alpha^{\prime}} \frac{1}{2 \gamma} \int_{r_{h}}^{\infty} \frac{\hat{c}_{3} d r}{\hat{c}_{2}^{2}\left(\hat{c}_{2}^{2}-\hat{c}_{1}^{2}\right)^{1 / 2}}, \quad \frac{L}{2}=\frac{1}{\gamma^{1 / 2}} \int_{r_{h}}^{\infty} \frac{\hat{c}_{3} d r}{\hat{c}_{2}^{2}\left(\hat{c}_{2}^{2}-\hat{c}_{1}^{2}\right)^{1 / 2}} .
$$

Notice that $S_{I} \propto L^{2}$, and the universality (or not) of the thermal Wilson loop expectation value (ans thus the jet quenching parameter) is related to the properties of the background integral $\mathcal{I}$

$$
\mathcal{I}=\int_{r_{h}}^{\infty} \frac{\hat{c}_{3} d r}{\hat{c}_{2}^{2}\left(\hat{c}_{2}^{2}-\hat{c}_{1}^{2}\right)^{1 / 2}}
$$

Further simplification is possible by noticing that reduction from the ten dimensional string frame (3.5) to the five-dimensional Einstein frame ${ }^{7}$ leads to

$$
\mathcal{I}=\int_{r_{h}}^{\infty} e^{-\Phi / 2} \Omega_{1}^{2 / 3} \Omega_{2}^{8 / 3} \frac{c_{3} d r}{c_{2}^{2}\left(c_{2}^{2}-c_{1}^{2}\right)^{1 / 2}}
$$

The utility of the five dimensional Einstein frame is because in all gauge theory-string theory dualities (subject to condition of [18]) one has

$$
\left[\frac{c_{2}^{4}}{c_{3}}\left[\frac{c_{1}}{c_{2}}\right]^{\prime}\right]^{\prime}=0
$$

\footnotetext{
${ }^{5}$ Given conformal invariance of the background geometry this is not surprising.

${ }^{6}$ This is obviously true for any asymptotically AdS or Klebanov-Tseytlin backgrounds.

${ }^{7}$ In our case the latter is simply $\left(-c_{1}^{2} d t^{2}+c_{2}^{2} \vec{x}^{2}+c_{3}^{2} d r^{2}\right)$.
} 
where derivatives are with respect to $r$. Of course, equation (3.17) can be verified explicitly in each example of duality — for the supergravity dual to cascading gauge theory it follows from eq.(32) and eq.(33) of [24]. Integrating (3.17) we find

$$
\left[\frac{c_{1}}{c_{2}}\right]^{\prime}=2 \pi T c_{2, h}^{3} \frac{c_{3}}{c_{2}^{4}}
$$

where $c_{2, h}=c_{2}\left(r_{h}\right)$. Now, (3.18) allows to change the integration variable in (3.16) from $r$ to $x$ given by (2.9), we find

$$
\mathcal{I}=\frac{1}{2 \pi T c_{2, h}^{3}} \int_{0}^{1} d x \frac{c_{2}(x)}{\sqrt{1-x^{2}}} e^{-\Phi(x) / 2} \Omega_{1}^{2 / 3}(x) \Omega_{2}^{8 / 3}(x) .
$$

In a class of gauge theory-string theory dualities discussed in [18] (and [20]) the part of the integrand in (3.19) depending on the five-dimensional metric warp factor $c_{2}$ and the dilaton $\Phi$ is universal; the remaining factor is $\propto g_{\perp}^{1 / 3}$, where $g_{\perp}$ is the determinant of the transverse (angular) Einstein frame metric ${ }^{8}$. Obviously, $\mathcal{I}$ is not "universal".

We conclude this section with explicit evaluation of the jet quenching parameter in cascading gauge theories. Using background metric (2.11) and (2.12) we find (to leading order in $\left.\delta_{\text {cascade }}\right)$

$$
\rho(T)=\frac{\hat{q}_{\text {cascade }}}{\hat{q}_{K W}}=1+\frac{P^{2}}{K_{*}} \chi+\mathcal{O}\left(\frac{P^{4}}{K_{*}^{2}}\right),
$$

with $^{9}$

$$
\chi=\frac{1}{2}+3 \xi(0)-\frac{\sqrt{2}\left(\Gamma\left(\frac{3}{4}\right)\right)^{2}}{6 \pi^{3 / 2}} \int_{0}^{1} d x \frac{6 \xi(x)+20 \eta(x)-3 \zeta(x)}{\left(1-x^{2}\right)^{3 / 4}} .
$$

Numerically evaluating the integral in $(3.21)$ we find

$$
\chi \approx-1.388
$$

Since $\chi<0$ and $K_{*}$ increases with temperature (2.1), we obtain (2.5).

\footnotetext{
${ }^{8}$ It is easy to reproduce $\mathcal{N}=4$ result of [1] from our general approach.

${ }^{9}$ In terms of supergravity parameters $\hat{q}_{K W}$ is identical to that of $\hat{q}_{\mathcal{N}=4}$ computed in [1]. However, these supergravity parameters have a different relation to gauge theory parameters in Klebanov-Witten and $\mathcal{N}=4$ superconformal gauge theories. It can be shown that $\frac{\hat{q}_{K W}}{\hat{q}_{\mathcal{N}}=4}=\sqrt{\frac{27}{32}}$, due to the difference in the $S^{5}$ and $T^{1,1}$ volumes and the fact that Klebanov-Witten superconformal gauge theory contains two gauge groups [38].
} 


\section{Jet quenching in QCD}

${ }^{10}$ As we stated in the introduction, asymptotically free gauge theories are not in the universality class of gauge theories dual to weakly coupled supergravity backgrounds. Nonetheless, it is interesting to see what would be the prediction of QCD jet quenching parameter $\hat{q}_{Q C D}$ from supergravity. We propose that such a relation could be obtained by expressing the temperature-dependent deviation of $\hat{q}_{\text {cascade }}$ from $\hat{q}_{\mathcal{N}=4}$ in terms of the temperature-dependent deviation of the speed of sound from the conformal result. Combining (3.20) and (2.3) we find

$$
\hat{q}_{\text {cascade }}=\hat{q}_{K W} \times\left(1+\frac{9 \chi}{4}\left(\frac{1}{3}-v_{s}^{2}\right)\right)
$$

Now, (4.1) could be adapted to real QCD replacing $\hat{q}_{\text {cascade }} \Rightarrow \hat{q}_{Q C D}, \hat{q}_{K W} \Rightarrow \hat{q}_{\mathcal{N}=4}$ and

$$
v_{s}^{2} \Rightarrow\left(v_{s}^{Q C D}\right)^{2}=\frac{\partial P_{Q C D}}{\partial \epsilon_{Q C D}}
$$

where $P_{Q C D}(T)$ and $\epsilon_{Q C D}(T)$ are correspondingly the pressure and the energy density of QCD plasma in the regime relevant at RHIC.

We expect that $\chi$ computed from the cascading gauge theory would differ from the corresponding coefficient extracted from $\mathcal{N}=2^{*}$ gauge theory plasma. It is thus interesting to obtain numerical value for $\hat{q}_{Q C D}$ from different dual supergravity models and see whether the result is robust.

\section{Acknowledgments}

I would like to thank Ofer Aharony, Hong Liu and Krishna Rajagopal for valuable comments. Research at Perimeter Institute is supported in part by funds from NSERC of Canada. I gratefully acknowledge support by NSERC Discovery grant.

\section{References}

[1] H. Liu, K. Rajagopal and U. A. Wiedemann, "Calculating the Jet Quenching Parameter from AdS/CFT," arXiv:hep-ph/0605178.

[2] J. Casalderrey-Solana and D. Teaney, "Heavy quark diffusion in strongly coupled N = 4 Yang Mills," arXiv:hep-ph/0605199.

\footnotetext{
${ }^{10}$ I would like to thank Krishna Rajagopal for insightful comments that led to this discussion.
} 
[3] C. P. Herzog, A. Karch, P. Kovtun, C. Kozcaz and L. G. Yaffe, "Energy loss of a heavy quark moving through $\mathrm{N}=4$ supersymmetric Yang-Mills plasma," arXiv:hep-th/0605158.

[4] E. Caceres and A. Guijosa, "On drag forces and jet quenching in strongly coupled plasmas," arXiv:hep-th/0606134.

[5] R. Baier, D. Schiff and B. G. Zakharov, "Energy loss in perturbative QCD," Ann. Rev. Nucl. Part. Sci. 50, 37 (2000) [arXiv:hep-ph/0002198].

[6] A. Kovner and U. A. Wiedemann, "Gluon radiation and parton energy loss," arXiv:hep-ph/0304151.

[7] M. Gyulassy, I. Vitev, X. N. Wang and B. W. Zhang, "Jet quenching and radiative energy loss in dense nuclear matter," arXiv:nucl-th/0302077.

[8] P. Jacobs and X. N. Wang, "Matter in extremis: Ultrarelativistic nuclear collisions at RHIC," Prog. Part. Nucl. Phys. 54, 443 (2005) [arXiv:hep-ph/0405125].

[9] B. G. Zakharov, "Radiative energy loss of high energy quarks in finite-size nuclear matter and quark-gluon plasma," JETP Lett. 65, 615 (1997) [arXiv:hep$\mathrm{ph} / 9704255]$.

[10] J. M. Maldacena, "The large $N$ limit of superconformal field theories and supergravity," Adv. Theor. Math. Phys. 2, 231 (1998) [Int. J. Theor. Phys. 38, 1113 (1999)] [arXiv:hep-th/9711200].

[11] O. Aharony, S. S. Gubser, J. M. Maldacena, H. Ooguri and Y. Oz, "Large N field theories, string theory and gravity," Phys. Rept. 323, 183 (2000) [arXiv:hepth/9905111].

[12] K. Pilch and N. P. Warner, "N = 2 supersymmetric RG flows and the IIB dilaton," Nucl. Phys. B 594, 209 (2001) [arXiv:hep-th/0004063].

[13] J. Polchinski and M. J. Strassler, "The string dual of a confining four-dimensional gauge theory," arXiv:hep-th/0003136.

[14] I. R. Klebanov and M. J. Strassler, "Supergravity and a confining gauge theory: Duality cascades and chiSB-resolution of naked singularities," JHEP 0008, 052 (2000) [arXiv:hep-th/0007191]. 
[15] J. M. Maldacena and C. Nunez, "Towards the large N limit of pure $\mathrm{N}=1$ super Yang Mills," Phys. Rev. Lett. 86, 588 (2001) [arXiv:hep-th/0008001].

[16] A. Karch and E. Katz, "Adding flavor to AdS/CFT," JHEP 0206, 043 (2002) [arXiv:hep-th/0205236].

[17] T. Sakai and S. Sugimoto, "Low energy hadron physics in holographic QCD," Prog. Theor. Phys. 113, 843 (2005) [arXiv:hep-th/0412141].

[18] A. Buchel and J. T. Liu, "Universality of the shear viscosity in supergravity," Phys. Rev. Lett. 93, 090602 (2004) [arXiv:hep-th/0311175].

[19] P. Kovtun, D. T. Son and A. O. Starinets, "Viscosity in strongly interacting quantum field theories from black hole physics," Phys. Rev. Lett. 94, 111601 (2005) [arXiv:hep-th/0405231].

[20] A. Buchel, "On universality of stress-energy tensor correlation functions in supergravity," Phys. Lett. B 609, 392 (2005) [arXiv:hep-th/0408095].

[21] A. Buchel, J. T. Liu and A. O. Starinets, "Coupling constant dependence of the shear viscosity in $\mathrm{N}=4$ supersymmetric Yang-Mills theory," Nucl. Phys. B 707, 56 (2005) [arXiv:hep-th/0406264].

[22] P. Benincasa and A. Buchel, "Transport properties of $\mathrm{N}=4$ supersymmetric YangMills theory at finite coupling," JHEP 0601, 103 (2006) [arXiv:hep-th/0510041].

[23] P. Benincasa, A. Buchel and A. O. Starinets, "Sound waves in strongly coupled non-conformal gauge theory plasma," Nucl. Phys. B 733, 160 (2006) [arXiv:hepth/0507026].

[24] A. Buchel, "Transport properties of cascading gauge theories," Phys. Rev. D 72, 106002 (2005) [arXiv:hep-th/0509083].

[25] P. Benincasa and A. Buchel, "Hydrodynamics of Sakai-Sugimoto model in the quenched approximation," arXiv:hep-th/0605076.

[26] A. Buchel, "Finite temperature resolution of the Klebanov-Tseytlin singularity," Nucl. Phys. B 600, 219 (2001) [arXiv:hep-th/0011146]. 
[27] A. Buchel, C. P. Herzog, I. R. Klebanov, L. A. Pando Zayas and A. A. Tseytlin, "Non-extremal gravity duals for fractional D3-branes on the conifold," JHEP 0104, 033 (2001) [arXiv:hep-th/0102105].

[28] S. S. Gubser, C. P. Herzog, I. R. Klebanov and A. A. Tseytlin, "Restoration of chiral symmetry: A supergravity perspective," JHEP 0105, 028 (2001) [arXiv:hepth/0102172].

[29] O. Aharony, A. Buchel and A. Yarom, "Holographic renormalization of cascading gauge theories," Phys. Rev. D 72, 066003 (2005) [arXiv:hep-th/0506002].

[30] A. Buchel and J. T. Liu, "Thermodynamics of the $\mathrm{N}=2^{*}$ flow," JHEP 0311, 031 (2003) [arXiv:hep-th/0305064].

[31] A. Buchel, "N = 2* hydrodynamics," Nucl. Phys. B 708, 451 (2005) [arXiv:hepth/0406200].

[32] I. R. Klebanov and E. Witten, "Superconformal field theory on threebranes at a Calabi-Yau singularity," Nucl. Phys. B 536, 199 (1998) [arXiv:hep-th/9807080].

[33] J. M. Maldacena, "Wilson loops in large N field theories," Phys. Rev. Lett. 80, 4859 (1998) [arXiv:hep-th/9803002].

[34] S. J. Rey and J. T. Yee, "Macroscopic strings as heavy quarks in large N gauge theory and anti-de Sitter supergravity," Eur. Phys. J. C 22, 379 (2001) [arXiv:hepth/9803001].

[35] S. J. Rey, S. Theisen and J. T. Yee, "Wilson-Polyakov loop at finite temperature in large N gauge theory and anti-de Sitter supergravity," Nucl. Phys. B 527, 171 (1998) [arXiv:hep-th/9803135].

[36] A. Brandhuber, N. Itzhaki, J. Sonnenschein and S. Yankielowicz, "Wilson loops in the large N limit at finite temperature," Phys. Lett. B 434, 36 (1998) [arXiv:hepth/9803137].

[37] J. Sonnenschein, "What does the string / gauge correspondence teach us about Wilson loops?," arXiv:hep-th/0003032.

[38] H. Liu, private communication. 